\title{
Therapeutische Erfahrungen über das Kochsche albumosefreie Tuberkulin.
}

\author{
Yon \\ Dr. 0. Ország, und \\ Abteilungs-Chefarzt \\ Dr. I. Spitzstein, \\ ehem. Assistenten.
}

Es ist in der Geschichte der Therapie ganz merkwürdig und sonderbar, dass man über den Wert des spezifischen Heilverfahrens bei der Lungentuberkulose selbst nach 20 Jahren kein einheitliches Urteil gebildet hat. Unzweifelhaft steht jedoch so viel fest, dass der grösste Teil der Kliniker ganz entschieden für die Tuberkulintherapie Stellung nahm und ihre Anwendung in geeigneten Fällen und bei richtiger und vorsichtiger Methodik in Verbindung mit anderen Heilmethoden für nötig hält. Durch das Streben, die Wirksamkeit der Tuberkulinpräparate $z u$ erhöhen und die unangenehmen Nebenwirkungen zu vermeiden, wurde eine ganze Reihe von Forschern zur Herstellung neuer Präparate veranlasst. In der Dresdener hygienischer Ausstellung waren ganze Reihen der Tuberkulin-Präparate sichtbar. An den meisten Anstalten wird jedoch hauptsächlich das Alttuberkulin $\mathrm{Kochs}$ angewendet, ausser diesem kommt noch meistens das Beraneksche Tuberkulin in Betracht. Und obzwar die spezifische Diagnostik und Therapie durch das alte Tuberkulin beherrscht war, war $\mathrm{Koch}$ selbst mit seinen Präparaten unzufrieden und trachtete dieselben selbst in seinen letzten Lebensjahren zu. vervollkommnen.

Das Tuberkulin gehört nämlich, wie bekannt, zu den stark wirkenden Mitteln und verursacht die unter dem Namen Reaktion bekannten unangenehmen Nebenerscheinungen. Die nicht ent- 
sprechende Dosierung kann auf den kranken Herd selbst derartig schädlich einwirken, dass die Behandlung eingestellt werden muss. In den letzten Jahren gelang es jedoch, die Tuberkulinschäden durch vorsichtige I)osierung, durch die Behandlung mit kleinen Mengen zu vermeiden. Koch schrieb dieselben den in seinem Präparate befindlichen Albumosen und Peptonen zu und trachtete cleren Wirkung bei der Tuberkulinbehandlung auszuschalten.

Das Entfernen der Albumosen aus dem Alttuberkulin ist von Gabrilowitz durch chemische Behandlung des TA. unternommen worden. Sein Präparat, das Tuberculinum purum oder Endotin erregte Zweifel bei mehreren Forschern in der Hinsicht, ob durch die chemische Entfernung der Albumosen nicht auch ein Teil des Tuberkulins Schaden erleidet. Fs sind daher verschiedene Ansichten über Endotin veröffentlicht worden.

Robert Koch trachtete das geschilderte Ziel auf der Weise zu erreichen, dass er den zur Herstellung des Tuberkulins nötigen Nährboden aus eiweissfreien und unschädlichen Stoffen zusammengestellt hat und hierdurch die Beimischung von Extraktirstoffen und Peptonen zu verhindern glaubte. Dieser Nährboden bestand aus Asparagin 0,5\%, Magnesium eitricum 0,25\%, Monokaliumphosphat $0,5 \%$, Magnesiumsulfat $0,060,0$, Glyzerin $20^{\circ}$, Soda $0,25 \%$, Aqua destillata ad 100. Es enthält daher an organischen Bestandteilen Asparagin, Glyzerin und Magnesium citricum. Die auf diesem Nährboden gewachsene Kultur wurde jedoch bei der Bereitung des Tuberkulins nicht so hochgradig erhitzt, wie es beim A. geschieht. Das neue Präparat dürfte daher auch solche spezifischen Stoffe enthalten, die bei höherer Temperatur zugrunde gehen. Das Tuberkulin wird auf das Viertel seines Originalvolumens eingeengt unter dem Namen TAF/albumosefrei durch die Höchster Fabrik in den Handel gebracht.

Mit der wissenschaftlichen Prüfung und Untersuchung des neuen Präparates befassten sich $\mathrm{J}$ och mann und M öllers in dem Koch schen Institut. Sie fanden es spezifisch, indem es tuberkulöse Meerschweinchen unter den charakteristischen Symptomen des Tuberkulintodes tötet und mit dem spezifische Antikörper enthaltenden Serum Tuberkulöser Komplementbindung ergibt. Es gibt des weiteren die Präzipitationsreaktion und ruft die kutane, subkutane und intrakutane Reaktion hervor. Nach der Einspritzung sind an dem Kranken Herderscheinungen wahrnehmbar. Das Präparat wird von $\mathrm{Joch}$ mall $n$ und Möllers zu Heilzwecken hauptsächlich deswegen empfohlen, da es milde und reaktionslose Kuren ermöglicht, wobei maxi- 
male Dosen in relativ kurzer Zeit zu erreichen sind. Sie empfelllon seine Anwendung dank der geringen Reaktionserscheinungen auch für die ambulante Praxis. Für die subkutane Tuberkulinprobe empfehlen sie es wegen der geringen subjektiven Erscheinungen. Nach Beobachtung der erwähnten Forscher wird die Empfindlichkeit des Organismus dem Alttuberkulin gegenüber durch die Behandlung mit TAF. nicht vermindert.

Auf der Tuberkuloseärzte-Versammlung in Dresden hat Z i e gler ganz, gleichlautende Erfahrungen vorgetragen; dem Vortragra folgte eine lebhafte Diskussion, in der clas neue Präparat grosse Anerkennung fand.

Wir wandten unsere Aufmerksamkeit seinem therapeutischen Werte zu. Die diagnostische Anwendung schien uns a priori nicht viel zu versprechen. Die Literatur über probatorische Alttuberkulininjektionen ist im Laufe der Jahre beträchtlich angewachsen. Es ist klarer geworden, dass der praktische Wert desselben für die Diagnose der Lungentuberkulose tatsächlich gering ist. Infolge der Vervollkommnung der Perkussion, der Anwendung der Schwellenwert-Perkussion sind wir auch seltener an das Tuberkulin gebunden. Für das Vorhandensein einer Lungenaffektion sind nur die nach der Injektion auftretenden Herderscheinungen beweisend, denn das Fieber allein kann auch bei klinisch Gesunden oder anderweitig Erkrankter. hervorgerufen werden, wie es z. B. bei Syphilis der Fall ist. In Anbetracht dessen, dass es nach mehrjährigen Untersuchungen noch lheute nicht mit Sicherheit festgestellt ist, welche Alttuberkulinmenge für die sichere Feststellung der Tuberkulose ausschlaggebend ist, können wir die Anwendung eines neuen Präparates noch weniger für zweckmässig halten, da der geänderte Tuberkulingehalt desselben eine ganze Reihe neuer Untersuchungen und Beobachtungen erfordern würde, ohne eigentlich bessere Resultate zu versprechen.

Die Kutanreaktion besitzt bei Erwachsenen keinen besonderen Wert. Bloss zu Vergleichszwecken haben wir Versuche mit einer 25\%igen Lösung von TAF. und TA. angestellt. In der Mehrzahl der Fälle sahen wir nach Anwendung des TA. stärkere Reaktionen, die sicherlich dem grösseren Tuberkulin- und nicht dem Albumosegehalt des Präparates zuzuschreiben sind. Es scheint uns unzweifelhaft, dass das TAF. bei der diagnostischen Bewertung der Pir quetschen Reaktion noch weniger in Betracht kommt wie das TA.

Vor Ausführung unserer Heilversuche mussten wir eine prinzipielle Frage erwägen, sollen wir die Behandlung auf grewohnter Weise mit kleinen Dosen oder, wie $\mathrm{Ziegler}$ es empfohlen, mit 
$1 \mathrm{mg}$ beginnen. Diese vielbestrittene Frage der Tuberkulinbehandlung scheint im Kreise der Alttuberkulin-Anhänger zugunsten der kleinen Dosen entschieden zu werden. Wir halten die Behandlung mit grösseren Dosen des TAF. nicht berechtigt, da es auch Fieberreaktionen zu verursachen imstande ist. Wir begannen aus Vorsicht die Behandlung mit $1 / 10000 \mathrm{mg}$ und sahen in mehreren Fällen Fieberreaktionen, ein Beweis für die Richtigkeit unserer Vorsicht. Die Wirkungsweise des Tuberkulins motiviert in vollem Masse die Behandlung mit kleinen Dosen. Man bezweckt durch das Tuberkulin einesteils die Giftangewöhnung, andererseits die Beeinflussung des Krankheitsherdes. Vom Standpunkte der Giftangewöhnung ist der Beginn mit kleinen Mengen und die allmähliche Steigerung als richtig zu betrachten. Die Angewöhnung an das einverleibte Tuberkulin ist jedoch nicht identiseh mit der an die in dem Organismus entstandenen Toxinen. Es sind ja gegen Tuberbulin immunisierte Individuen bekamnt, deren Krankheit unverändert besteht und deren Auswurf reichlich $\mathrm{Kochsche} \mathrm{Bakterien} \mathrm{enthält.}$

Der wichtigste therapeutische Moment, die Beeinflussung des kranken Herdes ist mit kleinen Dosen erreichbar. Eine derartige Behandlung ist zweckmässiger und motivierter, um so mehr, da man auf diese Art dem Patienten nicht schaden kann.

Einen Nachteil der Tuberkulintherapie bildet der Umstand, dass wir über: keine Methode verfügen, die die Kontrolle der entsprechenden Dosierung ermöglichen würde. Unsere Behandlungsweise ist also heute eine tastende, welche die starken Reaktionen möglichst $z u$ vermeiden trachtet.

Wir haben bis Abschluss dieser Arbeit 51 Patienten längere Zeit mit TAF. behandelt, darunter 29 Frauen und 22 Männer. Von den ersteren waren 6 im I., 19. im II. und 4 im III. Stadium. Von den Männern waren $4 \mathrm{im} \mathrm{1.,} 15 \mathrm{im}$ II. und $3 \mathrm{im}$ III. Stadium. Der grösste Teil der Patienten hatte subfebrile Temperaturon, stark Fiebernde wurden nur in spärlichen Fällen geimpft. Die Tuberkulinkur wurde erst nach einmonatiichem Aufenthalte des Patienten im Sanatorium begonnen, damit die Beurteilung des Resultates durch den Erfolg der hygieno-diätetischen Behandlung nach Möglichkeit nicht beeinflusst werde.

In einigen Fällen zeigten sich bereits nach kleinen TAF.-Mengen starke Reaktionen. Die Lokalisation war in der Form einer lebhaft roten, empfindlichen Papel eine recht häufige Erscheinung, die erst nach 3-4 Tagen zurückging, Fieberreaktionen wurden auch vach $1 / 10000 \mathrm{mg}$ beobachtet, mit Temperatursteigerungen bis auf $38,7^{0}$. 
Bei einem der Patienten sahen wir später nach $6 / 10000 \mathrm{mg}$ wiederholt Fieberreaktionen. Aber auch in diesen Fällen klagten die Patienten nicht so viel über Beschwerden, wie dies bei der Behandlung mit dem TA. oft der Fall ist. Das Allgemeinbefinden war zumeist gut, Mattigkeit, Kopfschmerz, Übelkeit wurde nicht erwähnt. Herderscheinungen wurden seltener beobachtet; wir erklärten dies mit der vorsichtigen Dosierung.

Bei der Bestimmung des Heilwertes legten wir auf die Veränderung des Lungenbefundes, des Auswurfs und der Temperatur besonderes Gewicht. Den schönsten Erfolg sahen wir in der Abnahme des Fiebers. Melrere Patienten sind vollkommen fieberfrei geworden. Bei Frauen war auffallend die Abkürzung der prämenstruellen Temperatursteigerungen. Wir hatten Fälle, wo vor der Menstruation 2 Wochen lang erhöhte Temperaturen bestanden, im Laufe der TAF.-Behandlung wurde ihre Dauer bedeutend geringer, in einigen Fällen schwanden sie sogar vollkommen. Schädliche Wirkungen des Präparates haben wir noch nicht gesehen. In einem Falle beobachteten wir zwar eine sich 8 Tage lang hinziehende Hämoptoe; ein direkter Zusammenhang konnte jedoch zwischen der Blutung und der Injektion nicht angenommen werden. Die stark fiebernde Patientin litt an einer fibrösen, zu Blutungen neigenden Phthise.

Nach den Untersuchungen von Jochmann und Möllers kann das TAF. die Empfindlichkeit des Organismus dem 'TA. gegenüber nur in geringem Grade herabsetzen. Dies wäre damit zu erklären, dass das TA. in dem TAF. nicht enthaltene, andere Toxine enthält, die bei der stärkeren Erhitzung der Kulturflüssigkeit gewonnen werden. Demgegenüber konnten wir auch beobachten, dass der Organismus nach längerer Behandlung mit TA. dem TAF. gegenüber empfindlich bleibt. So erhielt ein Patient. nach längere Zeit dauernder Immunisierung mit kleinen bis $4 / 100 \mathrm{mg}$ steigenden Dosen von TA. $1 / 10 \mathrm{mg}$ TAF., also eine solche Menge, mit der in Deutschland die Behandlung begonnen wird. In unserem Falle entstand eine sehr lebhafte Reaktion. In Anbetracht dessen, dass das weniger konzentrierte TAF. selbstredend weniger Tuberkulin enthält als das T'A., ist die Reaktion nur dadurch zu erklären, dass das TAF. auch andere Tuberkuline enthält wie das TA., gegen welches der Organismus nicht immunisiert ist. Für diese Erklärung spricht auch folgende Erfahrung: Der mit BeranekTuberkulin behandelter Patient bekommt eine TAF.-Injektion, ohne dass danach eine Reaktion zu beobachten wäre. Das Beraneksche 
Tuberkulin wird nämlich auf albumosearmem Nährboden hergestellt, womit eine ähnliche Wirksamkeit des TAF. und des Beranekschen Präparates zu erklären ist, wie auch die Verschiedenheit beider und des TA.

Es ist anzunehmen, dass die Wirkung der auf den verschiedenen Nährböden hergestellten Tuberkuline verschieden ist. Dies ist der Fall bei der Züchtung der verschiedenen anderen Bakterien; zur Herstellung der Toxine sind in der Regel stets bestimmte optimale Nährböden gebräuchlich. Unseres Erachtens unterscheidet sich also das TAF. von dem TA. nicht nur dadurch, dass es albumosefrei, weniger konzentriert und bei niedrigerer Temperatur hergestellt und weniger toxisch ist, sondern auch dadurch, ditss das auf albumosefreiem Nährboden hergestellte Tuberkulin auch qualitativ von dem TA. einigermassen verschieden ist.

Was nun die therapeutische Bedeutung des TAF. anbelangt, ist feststellbar, dass die meisten behandelten Patienten an Kraft und Gewicht zunahmen, das Fieber sank, in einigen Fällen ganz schwand. Der Bazillengehalt des Sputums hat sich in vielen Fällen vermindert, in manchen sind die Bazillen gänzlich verschwunden. Wir sahen aber auch solche hochfiebernde Fälle, in denen keino Besserung zu erzielen war. Bei der Beurteilung der Resultate muissen wir den Umstand in Betracht ziehen, dass unsere Kranken streng hygienisch-diätetisch behandelt wurden. Die Erfolge dieser Kuren sind schon lange an vielen mit Tuberkulin nicht behandelten Kranken erwiesen. Andererseits gestehen wir, dass unsere Patienten im allgemeinen relativ kurze Zeit behandelt worden sind. Dieselben sind nämlich meistens nicht in der Lage, längere Zeit in der Heilanstalt zu verweilen und konnten deswegen nur einige Monate spozifisch behandelt werden.

\section{Zusammenfassung.}

Die erwähnten Erfahrungen mit den früheren über TA. vergleichend, wollen wir in dieser Arbeit uns mit der Tuberkulintherapie nicht im allgemeinen und des näheren befassen. Wir begnügen uns festzustellen, dass es ohne Zweifel steht, dass das T'AF. in Verbindung mit der hygienisch-diätetischen Behandlung mindestens so gute Erfolge ergibt als das TA. Es hat den Vorteil, dass es Fieberreaktionen seltener hervorruft, und auch dann ohne unangenehmen Allgemeinerscheinungen, die nur selten und gering zu beobachten sind. Diese Eigenschaft macht es empfehlenswert, dass 
7] Therapeut. Erfahrungen über das Kochsche albumosefreie Tuberkulin. 541

das letzte Präparat Kochs eher zu Heilzwecken angewandt wird, als die übrigen älteren des berühmten Forschers 1 ).

\section{Literatur.}

Jochman und Möllers, Über die Behandlung der Tuberkulose mit Kochs albumosefreiem Tuberkulin. Veröff. der Koch-Stiftung 1912. 3.

Ziegler, Erfahrungen über das Koch sche albumosefreie Tuberkulin. VIII. Versammlung der Taberkuloseärzte 1911. Diskussion: Ebenda.

Froymuth, Brauers Beiträge z. Tub. 1911.

1) Die seit Abschluss dieser Arbeit beobachteten Fälle bekräftigen unsere früheren Wahrnehmungen. 